\title{
Hyperinsulinemia promotes metastasis to the lung in a mouse model of Her2-mediated breast cancer
}

\author{
Rosalyn D Ferguson, Emily J Gallagher, Dara Cohen, Aviva Tobin-Hess, \\ Nyosha Alikhani, Ruslan Novosyadlyy, Nadine Haddad, Shoshana Yakar \\ and Derek LeRoith
}

Division of Endocrinology, Diabetes and Bone Diseases, The Samuel Bronfman Department of Medicine, Mount Sinai School of Medicine, New York, New York 10029, USA
Correspondence should be addressed to $D$ LeRoith Email derek.leroith@mssm.edu

\begin{abstract}
The Her2 oncogene is expressed in $\sim 25 \%$ of human breast cancers and is associated with metastatic progression and poor outcome. Epidemiological studies report that breast cancer incidence and mortality rates are higher in women with type 2 diabetes. Here, we use a mouse model of Her2-mediated breast cancer on a background of hyperinsulinemia to determine how elevated circulating insulin levels affect Her2-mediated primary tumor growth and lung metastasis. Hyperinsulinemic $\left(\mathrm{MKR}^{+/+}\right)$mice were crossed with doxycycline-inducible Neu-NT (MTB/TAN) mice to produce the MTB/TAN/MKR ${ }^{+/+}$mouse model. Both MTB/TAN and MTB/TAN/MKR ${ }^{+/+}$mice were administered doxycycline in drinking water to induce Neu-NT mammary tumor formation. In tumor tissues removed at 2, 4, and 6 weeks of Neu-NT overexpression, we observed increased tumor mass and higher phosphorylation of the insulin receptor/IGF1 receptor, suggesting that activation of these receptors in conditions of hyperinsulinemia could contribute to the increased growth of mammary tumors. After 12 weeks on doxycycline, although no further increase in tumor weight was observed in MTB/TAN/MKR ${ }^{+/+}$compared with MTB/TAN mice, the number of lung metastases was significantly higher in $\mathrm{MTB} / \mathrm{TAN} / \mathrm{MKR}^{+/+}$mice compared with controls $\left(\mathrm{MTB} / \mathrm{TAN} / \mathrm{MKR}^{+/+} 16.41 \pm 4.18\right.$ vs MTB/TAN $5.36 \pm 2.72$ ). In tumors at the 6 -week time point, we observed an increase in vimentin, a cytoskeletal protein and marker of mesenchymal cells, associated with epithelial-to-mesenchymal transition and cancer-associated fibroblasts. We conclude that hyperinsulinemia in MTB/TAN/MKR ${ }^{+/+}$mice resulted in larger primary tumors, with more mesenchymal cells and therefore more aggressive tumors with more numerous pulmonary metastases.
\end{abstract}

\section{Key Words \\ - type 2 diabetes \\ - breast cancer}

\section{Introduction}

Over the last three decades, the relationship between type 2 diabetes and breast cancer has been evaluated by numerous epidemiological studies. Most of these support a positive association between diabetes and breast cancer, with several recent meta-analyses suggesting that women with type 2 diabetes are at significantly greater risk of 
developing, relapsing with, and dying from breast cancer than women who do not have diabetes (Larsson et al. 2007, Peairs et al. 2011, Boyle et al. 2012). The relationship between insulin, specifically, and breast cancer has been assessed by several epidemiological studies. Independent of all other confounding factors, hyperinsulinemia was reported to be a significant factor for incident breast cancer (Pisani 2008, Gunter et al. 2009), while another study reported an association between raised insulin levels and breast cancer metastasis and mortality (Goodwin et al. 2002). Both raised C-peptide (a marker of secreted insulin levels) and insulin resistance have been recently reported to be linked to breast cancer-specific death (Duggan et al. 2011, Irwin et al. 2011). High circulating insulin levels have also been associated with a risk of breast cancer recurrence (Formica et al. 2012). After breast tumor surgery, elevated insulin levels in the circulation have been reported to be associated with an adverse prognosis during the first 5 years after diagnosis (Goodwin et al. 2012). While epidemiological studies only suggest a link between hyperinsulinemia and accelerated tumor growth, some proof-of-concept experimental studies indicate that hyperinsulinemia indeed promotes tumor development (Fierz et al. 2010, Novosyadlyy et al. 2010).

In our previous studies, we have specifically focused on the impact of hyperinsulinemia on breast cancer progression using the MKR mouse model. Female MKR mice are insulin resistant and hyperinsulinemic while the remaining are nonobese and only mildly hyperglycemic. Using this hyperinsulinemic model, we have observed significantly increased growth of transgenic (PyVmT) and orthotopically induced mammary tumors and have demonstrated a mechanistic link between hyperinsulinemia and increased cancer progression through increased activation of the insulin receptor (IR)/IGF1 receptor (IGF1R) and the phosphatidylinositol 3-kinase (PI3-K)/Akt/mTOR pathway (Novosyadlyy et al. 2010).

In the clinical setting, the differential influence of type 2 diabetes and its complications on subtype-specific breast cancer warrants further investigation. Around 25\% of breast cancers belong to the subtype characterized by amplification of the gene encoding human epidermal growth factor receptor 2 (Her2), which is associated with high risk of metastasis and poor outcome (Slamon et al. 1989, Seshadri et al. 1993). Recently, a retrospective study has evaluated the benefit of the antidiabetic treatments metformin and thiazolidinediones on women with type 2 diabetes and Her $2+$ breast cancer. Initial analysis of the study cohort revealed that type 2 diabetes was a significant predictor of reduced overall survival in women with stage 2 or higher Her $2+$ breast cancer, independent of other confounding factors such as age, estrogen receptor (ER)/progesterone receptor (PR) expression, and BMI. Furthermore, the antidiabetic therapies significantly increased overall survival and significantly reduced the risk of Her2+ breast cancer-specific mortality (He et al. 2012). Although insulin levels in these patients were not reported, both metformin and thiazolidinediones are insulin sensitizers, suggesting that improvements in insulin resistance in type 2 diabetics could have an impact on Her $2+$ breast cancer progression.

Her2 (also known as ErbB2 or Neu in rodents) belongs to the epidermal growth factor receptor family of receptor tyrosine kinases (RTK), which includes Her1 (also known as EGFR or ErbB1), Her3 (or ErbB3), and Her4 (or ErbB4). Unlike the other family members, no specific ligand has been identified for Her2 and its activity is dependent on its dimerization with either ligand-activated EGFR or Her3. At high expression levels resulting from ERBB2 amplification, homodimers are also activated (Harari \& Yarden 2000, Yarden 2001). Despite the lack of kinase activity of Her3, dimers of Her2/Her3 constitute the most potent signaling combination of all EGFR family dimers (Pinkas-Kramarski et al. 1996, Holbro et al. 2003), and a prevention of Her2/Her3 heterodimerization provides a significant clinical benefit in patients with Her2+ breast cancer (Baselga \& Swain 2010). Activation of Her2/Her3 dimers leads to upregulation of multiple downstream pathways including the canonical PI3-K/Akt/mTOR signaling cascade and the mitogen-activated protein kinase (MAPK) pathway (Jin \& Esteva 2008). Importantly, these two pathways are also the principal signaling pathways involved in the growth-promoting effects of the activated IR/IGF1R in tumorigenesis. Indeed, Her2 also dimerizes with the IGF1R leading to the emergence of resistance to Her2 pharmacotherapies (Lu et al. 2001).

Transgenic mouse models of $\mathrm{Her} 2(\mathrm{Neu})$-mediated mammary carcinogenesis include those with constitutive activation of wild-type $\mathrm{Neu}(\mathrm{c}-\mathrm{Neu})$ or oncogenic (activated) Neu (Neu-NT) under control of the MMTV promoter. Both $\mathrm{c}-\mathrm{Neu}$ and $\mathrm{Neu}-\mathrm{NT}$ overexpression result in invasive mammary carcinomas with latency periods of around 7 and 3 months respectively (Ursini-Siegel $e t a l$. 2007). A conditionally activated model of mammaryspecific Neu-NT has also been engineered by crossing MMTV-reverse tetracycline transactivator (rtTA) (MTB) transgenic mice with mice bearing the TetO-Neu-NT transgene (TAN) to generate MTB/TAN offspring (Moody et al. 2002). Tumor latency in this model is short, with development of multiple mammary tumors with $100 \%$

Published by Bioscientifica Ltd. 
penetrance within a few weeks and further progression to spontaneous lung metastasis (Moody et al. 2002).

Given that in mammary tumors from Neu-NT transgenic mice, the PI3-K/Akt/mTOR signaling pathway should already be active, an interesting question that arises is whether the induction of systemic hyperinsulinemia in these mice could enhance Neu-NT-mediated tumor growth via IR/IGF1R activation. To address this issue, we crossed homozygous $\mathrm{MKR}^{+/+}$mice with MTB/TAN mice to yield $\mathrm{MTB} / \mathrm{TAN} / \mathrm{MKR}^{+/+}$offspring, thus generating hyperinsulinemic mice expressing a doxycycline-inducible Neu-NT transgene. When induced with doxycycline, MTB/TAN/MKR ${ }^{+/+}$mice develop early Neu-NT-mediated mammary gland hyperplastic changes more rapidly than controls and go on to develop larger mammary tumors. Furthermore, MTB/TAN/MKR ${ }^{+/+}$mice exhibit higher numbers of lung macrometastases, suggesting that chronic hyperinsulinemia can augment Neu-NT-mediated primary tumor growth as well as the progression to lung metastasis.

\section{Materials and methods}

\section{Animal studies}

Animal care and maintenance were provided by the Mount Sinai School of Medicine AAALAC Accredited Animal Facility. All procedures were approved by the Institutional Animal Care and Use Committee of the Mount Sinai School of Medicine according to the National Institute of Health Guidelines. All mice used in this study were on Friend Virus B (National Institute of health) $(\mathrm{FVB} / \mathrm{N})$ genetic background. Mice were housed four per cage in a clean mouse facility and fed a standard mouse chow (PicoLab Rodent Diet 20, 5053; LabDiet, Brentwood, MO, USA) ad libitum on a $12 \mathrm{~h}$ light: $12 \mathrm{~h}$ darkness cycle. Plasma insulin levels were measured by the sensitive rat insulin RIA kit (Millipore, St Charles, MO, USA). An insulin tolerance test was performed on animals previously fasted for $4 \mathrm{~h}$. Insulin $(0.75 \mathrm{units} / \mathrm{kg}$ of body weight) was injected intraperitoneally and blood glucose values were measured immediately before and 15,30 , and $60 \mathrm{~min}$ after insulin injection. For induction of $\mathrm{Neu}-\mathrm{NT}$, mice were administered $1.5 \mathrm{mg} / \mathrm{ml}$ doxycycline (SigmaAldrich) in drinking water for the duration of the period of tumor growth from 2 weeks up to 12 weeks. Doxycycline water was changed twice per week. Mice were followed on a daily basis and body score conditions were recorded. To determine tumor mass, each animal was killed and mammary tumors from all thoracic and inguinal glands were carefully dissected and weighed. For analysis of pulmonary metastases, mice were killed and lungs were inflated via the trachea with $10 \%$ formalin, removed, and examined for macrometastatic lesions.

\section{Mammary gland whole mount analysis}

Inguinal mammary glands were removed, placed on a glass slide, and fixed for $4 \mathrm{~h}$ in Carnoy's fixative $(60 \%$ ethanol (100\%), 30\% chloroform, and 10\% glacial acetic acid). Glands were serially hydrated in 100, 95, 70, 50, and $30 \%$ ethanol for $15 \mathrm{~min}$ each, rinsed in water for $5 \mathrm{~min}$, and stained overnight with carmine alum. Glands were then serially dehydrated in 30, 50, 70, 95, and $100 \%$ ethanol for 15 min each and cleared overnight in xylene. Glands were then covered by Mount-Quick mounting medium (Daido Sangyo, Tokyo, Japan), before a glass coverslip was placed on top. Photographs were carried out using a stereomicroscope (Zeiss, Thornwood, NY, USA) at $4 \times$ magnification. Quantification of the relative area of end buds was performed using Image $J$ by measuring the mean end bud area of four random images at $4 \times$ objective taken from each whole mount.

\section{Histology and immunofluorescence}

Lungs were fixed in 10\% formalin before being embedded in paraffin and sectioned and stained using hematoxylin and eosin (H\&E). For immunofluorescence studies, mammary tumors were cut in cross section at the time of killing, fixed in 10\% formalin before being embedded in paraffin, and sectioned. Five-micron sections were deparaffinized, rehydrated, and subjected to antigen retrieval. Primary antibodies used were a rabbit polyclonal antibody to vimentin (Cell Signaling Technologies, Danvers, MA, USA) and a mouse MAB to Neu (Abcam, Cambridge, MA, USA). Secondary antibodies used were AlexaFluor-568-conjugated goat anti-rabbit IgG and Alexa-Fluor-488-conjugated goat anti-mouse IgG (Invitrogen, Molecular Probes, Eugene, OR, USA). Nuclei were counterstained with $0.2 \mu \mathrm{g} / \mathrm{ml} 4^{\prime}$,6-diamidino2-phenylindole (DAPI; Sigma-Aldrich).

\section{Western blotting}

Tumor tissues were lysed in chilled lysis buffer ( $\mathrm{pH}$ 7.4) containing $50 \mathrm{mM}$ Tris, $150 \mathrm{mM} \mathrm{NaCl}, 1 \mathrm{mM}$ EDTA, $1.25 \%$ CHAPS, $1 \mathrm{mM}$ sodium orthovanadate, $10 \mathrm{mM}$ sodium pyrophosphate, $8 \mathrm{mM}$ B-glycerophosphate, and Complete Protease Inhibitor Cocktail tablet. Protein

Published by Bioscientifica Ltd. 
concentration of samples was measured using the BCA protein assay kit (Thermo Scientific, Rockford, IL, USA). Protein samples were resuspended in $3 \times$ loading buffer containing DTT (Cell Signaling Technologies) and denatured by boiling for $5 \mathrm{~min}$ at $96^{\circ} \mathrm{C}$. Samples were then subjected to SDS-PAGE ( 8 or 8-16\% Tris-glycine gel; Life Technologies) and transferred to a nitrocellulose membrane. Membranes were probed with the appropriate primary antibodies: anti-phospho IR- $\beta^{(\mathrm{Y} 1150 / 51)} / \mathrm{IGF} 1 \mathrm{R} \beta^{(\mathrm{Y} 1135 / 36)}$, anti-phospho $\mathrm{Akt}{ }^{\mathrm{Ser} 473}$, anti-total Akt, and anti-vimentin (Cell Signaling Technologies) and then reblotted with B-actin (Sigma-Aldrich) or anti-IR $\beta$ (Santa Cruz Biotechnology) before being incubated with secondary antibodies (LI-COR Biosciences, Lincoln, NE, USA) and being exposed to the LI-COR infrared detection system (LI-COR Biosciences).

\section{Statistical analysis}

Statistical analyses were conducted using the Student's $t$-test. Results are expressed as means \pm s.E.m.

\section{Results}

MTB/TAN/MKR ${ }^{+/+}$mice express the same metabolic phenotype as the parental $\mathrm{MKR}^{+/+}$mouse strain

Transgenic MMTV-rtTA (MTB) and TetO-Neu-NT (TAN) mice were previously crossed to yield bitransgenic MTB/TAN offspring demonstrating doxycycline-dependent Neu-NT expression in luminal mammary epithelial cells and subsequent development of gross mammary tumors and pulmonary macrometastases (Moody et al. 2002). We crossed MTB/TAN with homozygous MKR mice to yield $\mathrm{MTB} / \mathrm{TAN} / \mathrm{MKR}^{+/+}$mice. Female $\mathrm{MTB} / \mathrm{TAN} / \mathrm{MKR}^{+1+}$ mice showed the same metabolic abnormalities as the homozygous female $\mathrm{MKR}^{+/+}$mice (Novosyadlyy et al. 2010) namely lowered body weight, mild hyperglycemia, increased systemic insulin, and severe insulin resistance (Fig. 1A, B, C, and D).

\section{MTB/TAN/MKR ${ }^{+/+}$mice show augmented Neu-NT-induced abnormalities of the mammary gland at 8 weeks compared with MTB/TAN controls}

Others have shown that doxycycline-induced Neu-NT expression results in noticeable hyperplastic abnormalities in the mouse mammary gland such as the presence of cellular masses along the length of ducts, as well as terminal end bud enlargement due to the presence of
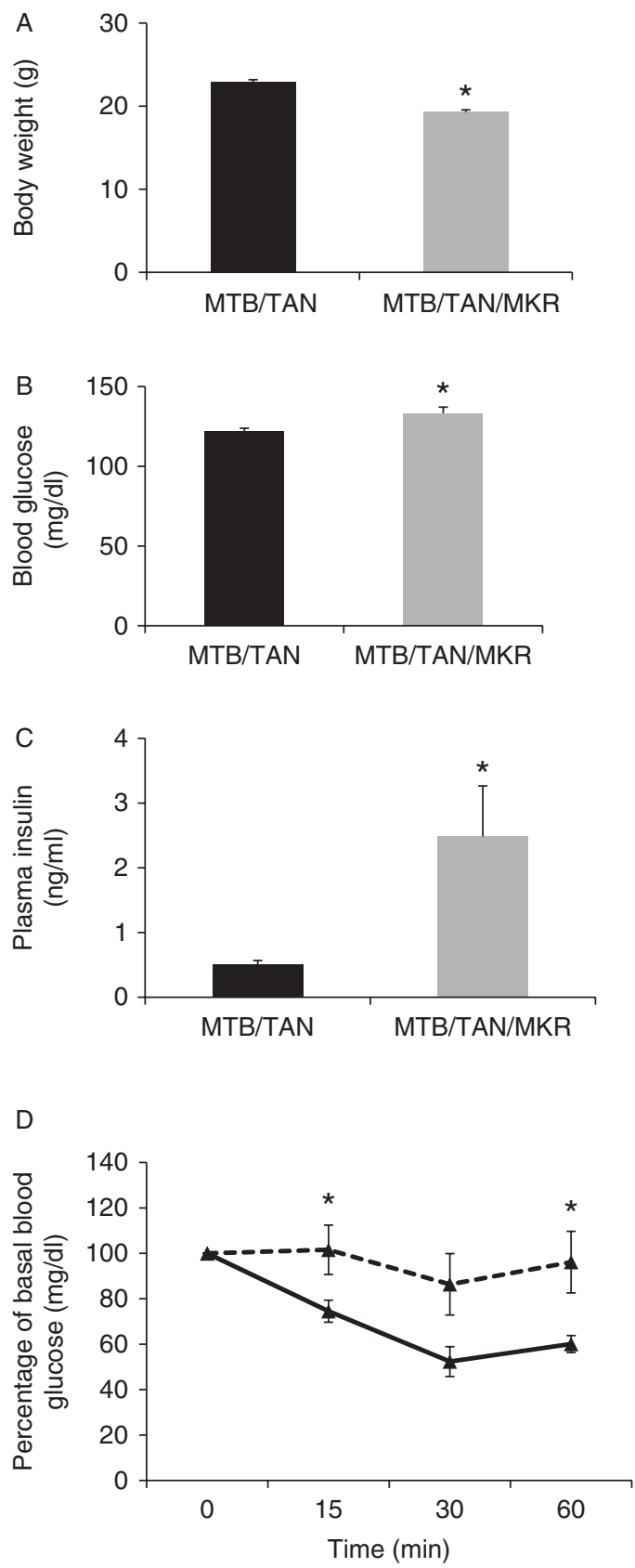

Figure 1

Metabolic characterization of the MTB/TAN/MKR ${ }^{+/+}$mouse model. (A) Body weight (MTB/TAN, $n=14$; MTB/TAN/MKR $\left.{ }^{+1+}, n=10\right)$, (B) blood glucose (MTB/TAN, $n=30$; MTB/TAN/MKR ${ }^{+/+}, n=22$ ), and (C) serum insulin (MTB/TAN, $n=13$; MTB/TAN/MKR ${ }^{+/+}, n=20$ ) of MTB/TAN and $\mathrm{MTB} / \mathrm{TAN} / \mathrm{MKR}^{+/+}$mice at 8 weeks of age. (D) An insulin tolerance test was performed on fasted 8-week-old MTB/TAN/MKR ${ }^{+/+}(n=5)$ and MTB/TAN mice $(n=5)$ after i.p. injection of insulin $(0.75$ units $/ \mathrm{kg})$. Blood samples were obtained from the tail vein and glucose concentrations were determined at the indicated time points. Graphs represent mean values of each group, error bars represent the S.E.M. ${ }^{*} P$ value $<0.05$.

Published by Bioscientifica Ltd 
acinar-like structures (Moody et al. 2002). We investigated whether hyperinsulinemia would have an additive effect on these early Neu-NT-mediated hyperplastic abnormalities by induced Neu-NT expression for 3 days in 8-week-old MTB/TAN/MKR ${ }^{+/+}$and MTB/TAN mice, killing the animals and comparing mammary gland morphology by whole mount analysis. As $\mathrm{MKR}^{+/+}$mice have precocious mammary gland growth and differentiation compared with control mice at both 3 and 15 weeks (Novosyadlyy \& LeRoith 2010), we also performed whole mount analysis and H\&E staining of mammary glands from 8-week-old MTB/TAN/MKR ${ }^{+/+}$and MTB/TAN mice, which had not been administered doxycycline (Fig. 2A and B, upper panels). In MTB/TAN/MKR ${ }^{+/+}$mice, we found Neu-NT-mediated abnormalities to be enhanced compared with MTB/TAN controls, with larger cellular masses growing on ducts, greater terminal end bud enlargement, and precocious lobular development (Fig. 2A, lower panels and C). This finding was further confirmed by histological evaluation of H\&E-stained

sections of the mammary gland (Fig. 2B, lower panels) where we observed advanced ductal hyperplasia in MTB/ TAN/MKR ${ }^{+/+}$mice compared with MTB/TAN controls.

\section{Neu-NT-induced mammary tumor burden is greater in MTB/TAN/MKR ${ }^{+/+}$mice than in MTB/TAN mice and is regulated through IR/IGF1R signaling pathways}

When doxycycline was administered for several weeks, MTB/TAN mice developed multiple invasive mammary adenocarcinomas with tumors arising in all inguinal and thoracic glands (Moody et al. 2002). We administered doxycycline to 8-week-old MTB/TAN and MTB/TAN/MKR ${ }^{+/+}$mice for $2,4,6$, or 12 weeks. Mice were killed at each time point and the combined tumor weight from all inguinal and thoracic glands from each mouse was recorded. As shown in Fig. 3A, MTB/ TAN/MKR ${ }^{+/+}$mice exhibited significantly higher total tumor mass than MTB/TAN controls at all time points with the exception of the 12-week time point, suggesting
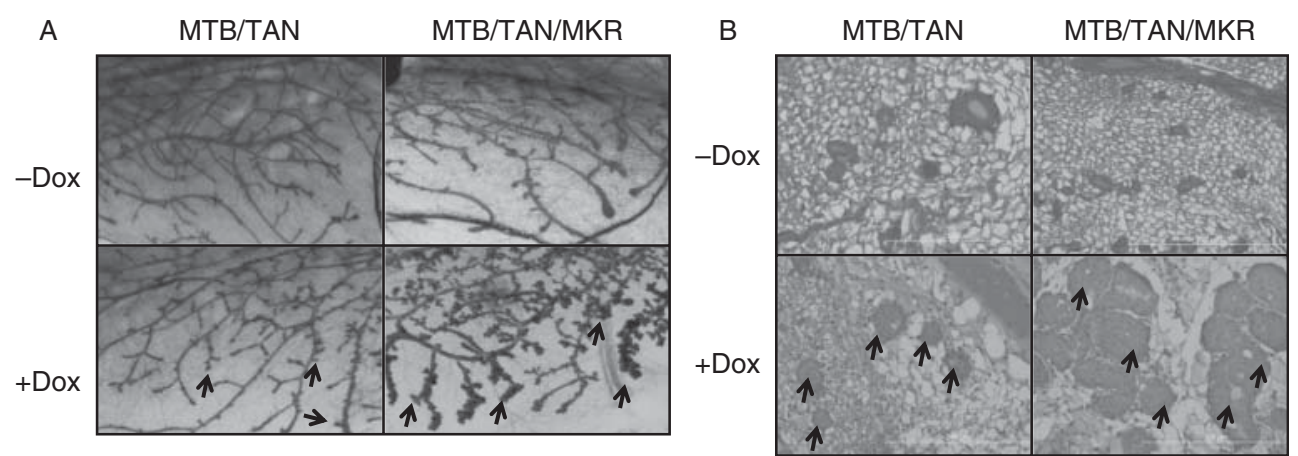

C

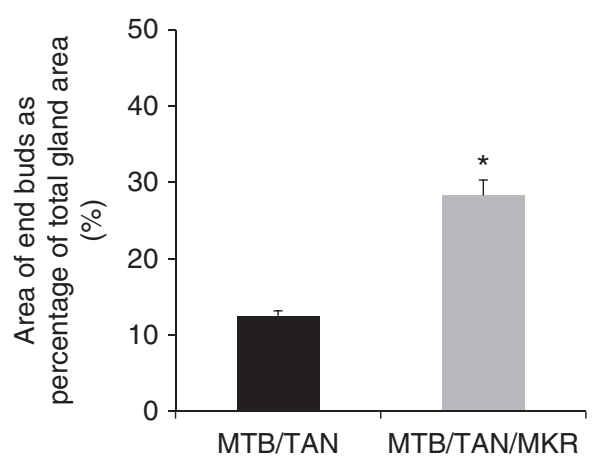

Figure 2

Effect of hyperinsulinemia on Neu-NT-mediated mammary gland hyperplasia. (A) Whole mount analysis of mammary glands obtained from 8-week-old female MTB/TAN and MTB/TAN/MKR ${ }^{+/+}$mice without doxycycline ( - Dox) and after 3 days of doxycycline treatment (+Dox). (B) Histological analysis (H\&E staining) of mammary glands after 3 days of doxycycline treatment. (C) Quantification of percentage area of mammary gland whole mounts composed of end buds (MTB/TAN, $n=3$; MTB/TAN/MKR, $n=3$ ). Arrows, End buds (A) and ductal hyperplasia. (C) At least five animals per group were analyzed and the representative images are shown. Original magnification, $\times 4$ (A) and $\times 40$ (B). Graph represents mean for each group, error bars represent s.E.M. $* P$ value $<0.05$ http://erc.endocrinology-journals.org DOI: 10.1530/ERC-12-0333
(C) 2013 Society for Endocrinology Printed in Great Britain 


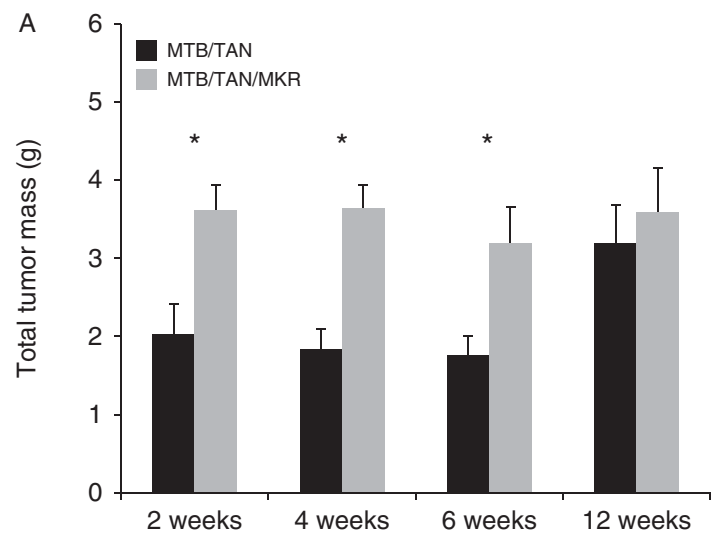

D

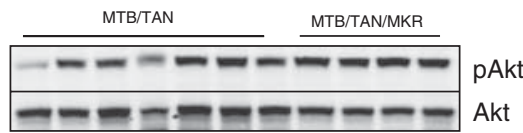

B
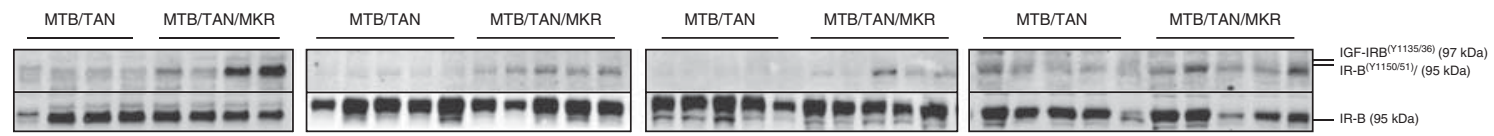

C
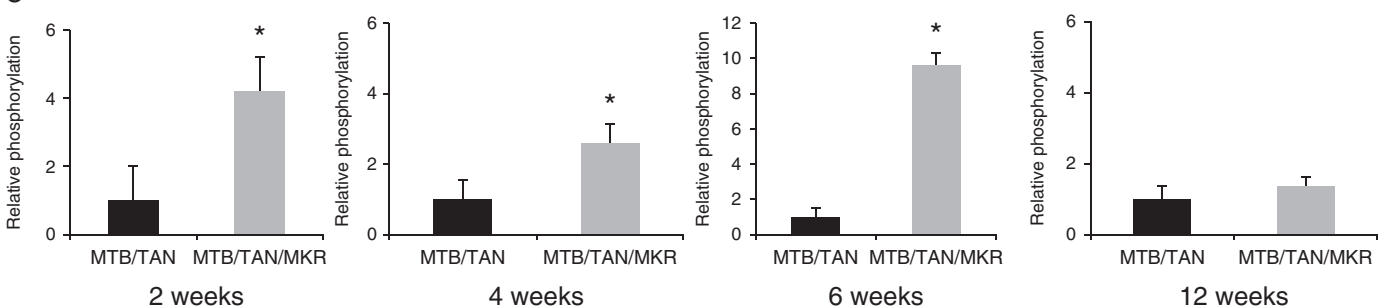

Figure 3

Tumor growth, IR $\beta / I G F 1 R \beta$, and Akt signaling are enhanced in tumors from MTB/TAN/MKR ${ }^{+1+}$ mice compared with MTB/TAN controls. (A) MTB/TAN and MTB/TAN/MKR ${ }^{+/+}$mice were maintained on doxycycline for 2, 4, 6, or 12 weeks. Mice were killed and the combined tumor mass from all mammary glands was recorded. For the 2-week time point, MTB/TAN, $n=11$; MTB/TAN/MKR ${ }^{+l+}, n=4$. For the 4-week time point, MTB/TAN, $n=11$; $\mathrm{MTB} / \mathrm{TAN} / \mathrm{MKR}^{+1+}, n=7$. For the 6 -week time point, MTB/TAN, $n=16$; $\mathrm{MTB} / \mathrm{TAN} / \mathrm{MKR}^{+1+}, n=6$. For the 12 -week time point, MTB/TAN, $n=12$; MTB/TAN/MKR ${ }^{+/+}, n=11$. (B) Tumor tissue was subjected to western blot

that hyperinsulinemia augments Neu-NT-driven mammary tumor growth. We extracted proteins from tumor tissues to examine whether IR/IGF1R activation was upregulated in $\mathrm{MTB} / \mathrm{TAN} / \mathrm{MKR}^{+/+}$mice compared with MTB/TAN controls. As shown in Fig. $3 \mathrm{~B}$ and $\mathrm{C}$, mammary tumor tissues from $\mathrm{MTB} / \mathrm{TAN} / \mathrm{MKR}^{+/+}$mice demonstrated higher levels of phosphorylated IR/IGF1R (IR $\beta /$ IGF1R $\beta$ ) after 2, 4, and 6 weeks of Neu-NT upregulation, suggesting that these receptors may be involved in mediating the additional tumor growth present in MTB/TAN/MKR ${ }^{+/+}$ mice. In MTB/TAN/MKR ${ }^{+/+}$and MTB/TAN mice that had been administered doxycycline for a period of 12 weeks, we observed an increase in IR/IGF1R phosphorylation in mammary tissues of $\mathrm{MTB} / \mathrm{TAN} / \mathrm{MKR}^{+/+}$mice, which was not statistically significant (Fig. $3 \mathrm{~B}$ and $\mathrm{C}$ ). There was no significant difference in tumor weights after 12 weeks analysis and probed with antibodies to phosphorylated $\operatorname{IR} \beta^{(\mathrm{Y} 1150 / 51)} /$ IGF1R $\beta^{(\mathrm{Y1135/36)}}$ and then reblotted with antibodies to IR $\beta$. (D) Western blot analysis of tumor tissue from mice maintained on doxycycline for 2 weeks probed with antibodies to phosphorylated Akt ${ }^{\text {Ser473 }}$ and total Akt. Using densitometry, protein expression was quantified ( $C$ and E). Error bars represent S.E.M. ${ }^{\star} P<0.05$. For the 2 -week time point, western blot is representative of MTB/TAN, $n=9$; MTB/TAN/MKR ${ }^{+1+}, n=4$ samples. For 4- and 6-week time points, MTB/TAN, $n=7 ; \mathrm{MTB} / \mathrm{TAN} / \mathrm{MKR}^{+1+}, n=7$

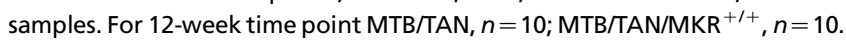

of doxycycline administration, possibly due to tumors reaching their physiologically maximal size after this time period (Fig. 3A). We also compared activation of Akt, which lies downstream of both the IR/IGF1R and Her2 and found that at 2 weeks there was a significant upregulation of phosphorylated Akt in tumors from MTB/TAN/MKR ${ }^{+/+}$mice compared with MTB/TAN mice (Fig. 3D and E). At later stages of tumor development, levels of activation of Akt in the tumors appeared to be similar in the two groups.

\section{Neu-NT-induced spontaneous lung macrometastases are enhanced in MTB/TAN/MKR ${ }^{+/+}$mice after 12 weeks of tumor growth}

MTB/TAN mice were reported to harbor Neu-NT-dependent spontaneous lung macrometastases (Moody et al. 2002). 
We removed the lungs of killed MTB/TAN/MKR ${ }^{+/+}$and MTB/TAN mice after 2, 4, 6, or 12 weeks of doxycyclineinduced $\mathrm{Neu}-\mathrm{NT}$ expression in mammary epithelium and recorded the numbers of visible macrometastases. We also examined H\&E-stained sections of lung at all time points for both micrometastases and macrometastases. After 2, 4, or 6 weeks of $\mathrm{Neu}$ expression and mammary tumor growth, no visible macrometastases were observed in either MTB/TAN/MKR ${ }^{+/+}$or MTB/TAN mice. However, H\&E staining revealed the presence of micrometastases in lungs of both groups of mice after 6 weeks of $\mathrm{Neu}$ expression (data not shown). Although there was a trend for the number of micrometastases to be increased in MTB/TAN/MKR ${ }^{+/+}$mice $(0.3$ micromets/ lung section in MTB/TAN vs 0.8 micromets/lung section in $\mathrm{MTB} / \mathrm{TAN} / \mathrm{MKR}^{+/+}$), this did not reach statistical significance. In contrast, after 12 weeks of $\mathrm{Neu}$ expression in mammary tissue, macrometastases could be clearly observed in the lungs, and these were significantly increased in MTB/TAN/MKR ${ }^{+/+}$compared with MTB/TAN mice (Fig. 4A and B), suggesting that hyperinsulinemia increases the metastatic potential of $\mathrm{Neu}$-driven mammary carcinogenesis.

\section{Neu-NT-induced mammary tumors in MTB/TAN/MKR ${ }^{+/+}$ mice express higher levels of vimentin}

To determine whether hyperinsulinemia enhances the progression of Neu-NT-mediated primary tumors to lung metastases, we analyzed tumor tissue for the expression of vimentin protein, a marker of mesenchymal cells. As shown in Fig. 5A and B, western blot analysis of tumor

A

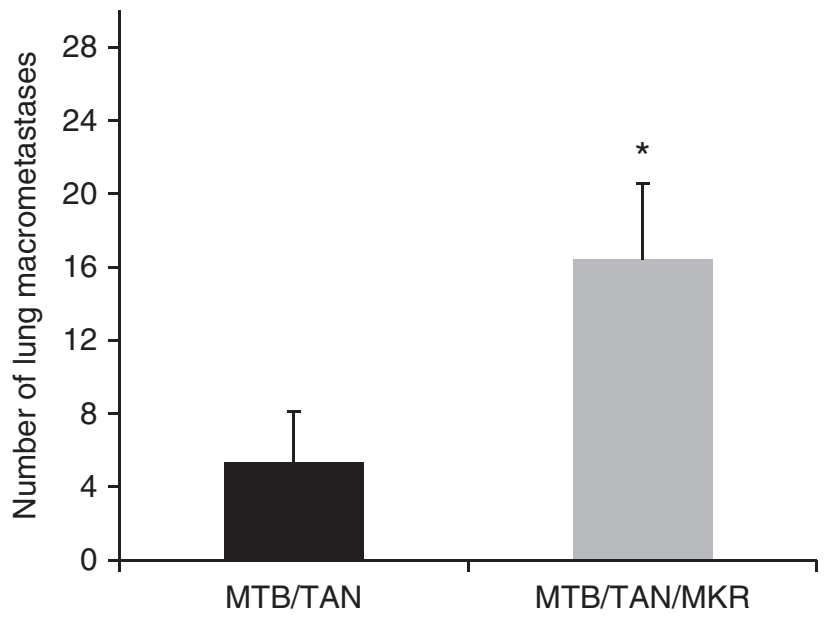

B

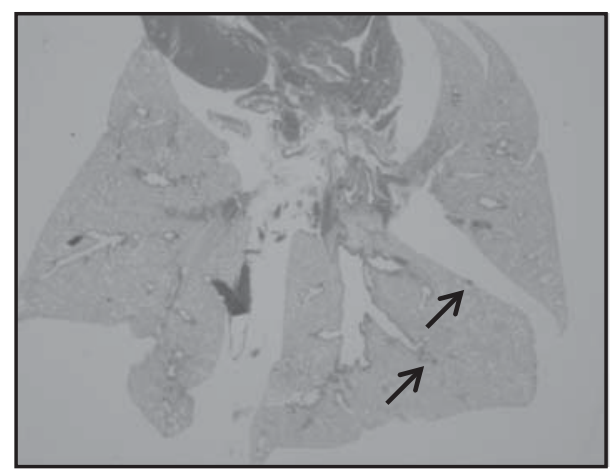

MTB/TAN

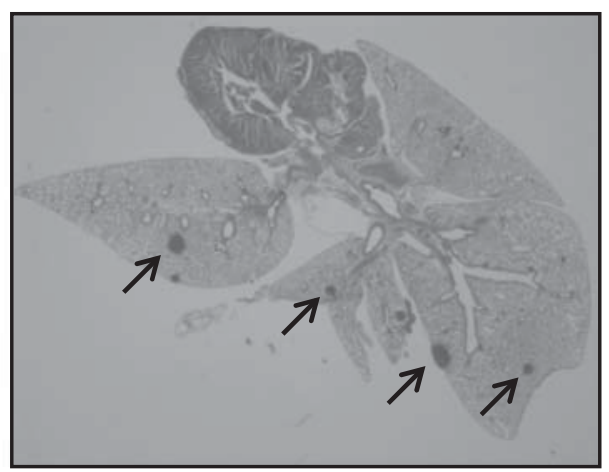

MTB/TAN/MKR

\section{Figure 4}

Metastatic progression is enhanced in MTB/TAN/MKR ${ }^{+/+}$mice. After 12 weeks on doxycycline, MTB/TAN and MTB/TAN/MKR ${ }^{+/+}$mice were killed, lungs were removed and inflated, and $(A)$ number of lung macrometastases were recorded. Graph represents the mean for each group, error bars represent S.E.M. ${ }^{*} P$ value $<0.05$. (B) Lungs were paraffin embedded, sectioned, and stained with $\mathrm{H} \& \mathrm{E}$ to reveal macrometastases. Original magnification $4 \times$. Arrows indicate metastatic cells.
(C) 2013 Society for Endocrinology Printed in Great Britain 
lysates demonstrated increased vimentin expression in tumors from 6-week-old MTB/TAN/MKR ${ }^{+/+}$mice compared with MTB/TAN mice. We additionally analyzed vimentin expression in 6-week tumor tissues by immunofluorescent staining of paraffin-embedded sections. As shown in Fig. 5B and C, vimentin levels as measured by immunofluoresence were significantly elevated, indicating that more mesenchymal cells were present in $\mathrm{MTB} / \mathrm{TAN} / \mathrm{MKR}^{+/+}$mice at this time point. The vimentinpositive cells generally did not stain positive for $\mathrm{Neu}$, indicating that these were either cells that had undergone epithelial-to-mesenchymal transition (EMT) and lost Neu expression, or were cancer-associated fibroblasts. At 4 and 12 weeks of tumor development, we did not observe significant differences in vimentin expression (data not shown).

A

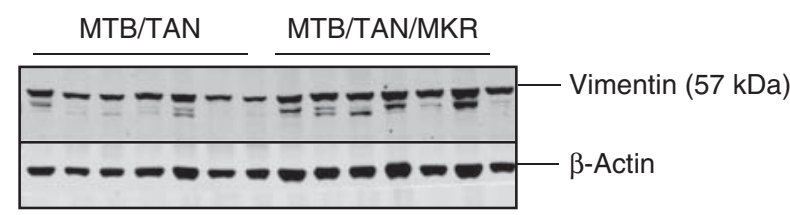

C
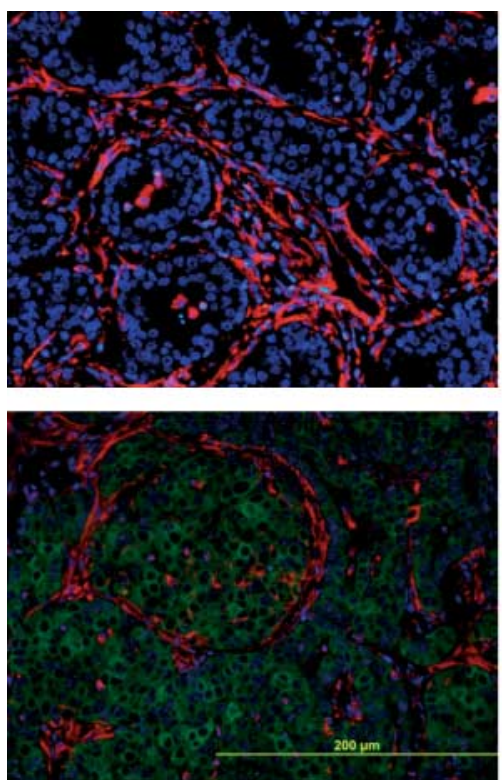

MTB/TAN
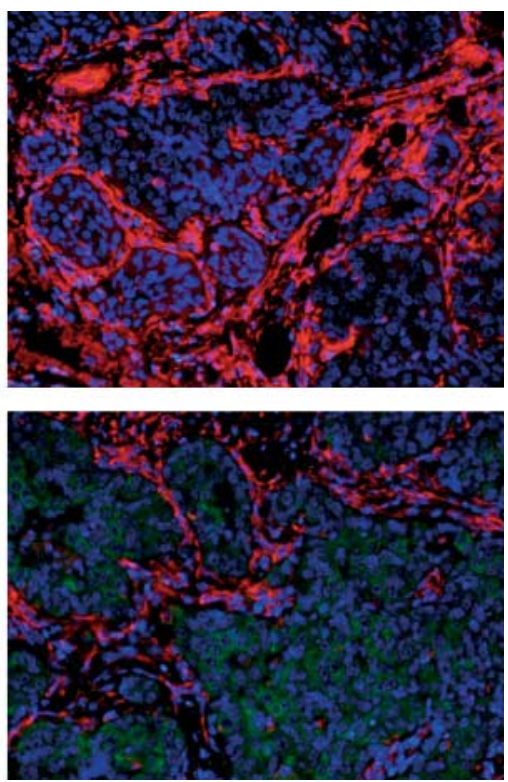

MTB/TAN/MKR

\section{Discussion}

Epidemiological evidence suggests a causal relationship between type 2 diabetes and the incidence, recurrence, and mortality from breast cancer. Hyperinsulinemia has been identified as a specific factor that may drive breast cancer cell proliferation, but data on human patients that focus on how specific subtypes of breast cancer respond to elevated circulating insulin are limited. Overexpression of ErbB2/Her2 is responsible for around 25\% of all human breast cancers and results in aggressive primary tumors, which commonly metastasize to the lungs. In this study, we have employed the MKR mouse model with a conditionally activated Neu-NT to represent this subtype of breast cancer. We have demonstrated for the first time

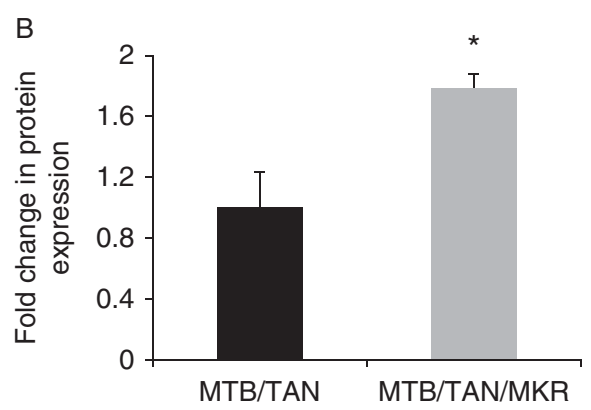

$\mathrm{D}$

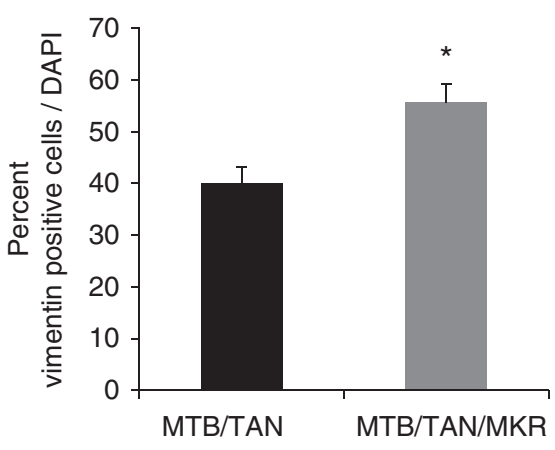

\section{Figure 5}

Vimentin expression is enhanced in MTB/TAN/MKR ${ }^{+/+}$mice. (A). After 6-weeks Neu-NT upregulation, mammary tumors were removed from MTB/TAN and MTB/TAN/MKR ${ }^{+/+}$mice, tumor lysates were subjected to western blot analysis, and probed with anti-vimentin antibodies. Using densitometry, protein expression was quantified (B). Tumor tissue was paraffin embedded, sectioned, and analyzed by immunofluorescence microscopy for the expression of vimentin (red) and Neu (green) proteins, nuclei were stained with DAPI (blue) (C). Vimentin expression was analyzed using 'Image $J$ ' program to quantify expression of vimentin protein in 6-week tumors from MTB/TAN and MTB/TAN/MKR ${ }^{+/+}$mice (D). Photographs are representative of at least four mice from each group. Five highpower fields were photographed on every slide and each image was quantified. Original magnification $40 \times$. Graphs represent the mean for each group, error bars represent S.E.M. ${ }^{*} P$ value $<0.05$. http://erc.endocrinology-journals.org DOI: 10.1530/ERC-12-0333 (c) 2013 Society for Endocrinology Printed in Great Britain 
in an animal model that hyperinsulinemia significantly affects the rate of Neu-NT-mediated mammary tumor development as well as the progression of breast cancer metastasis to the lungs.

Previously, we have employed the MKR mouse model to demonstrate that PyVmT-driven mammary gland hyperplasia at 3 and 15 weeks' postnatal development is more advanced under conditions of hyperinsulinemia (Novosyadlyy et al. 2010). Hyperplasia of the mammary gland has previously been shown to occur rapidly (3-4 days) after conditionally activated Neu-NT expression (Moody et al. 2002). In MTB/TAN/MKR ${ }^{+/+}$mice, we observed that in the presence of elevated insulin, mammary gland hyperplasia was more advanced after 3 days, suggesting that hyperinsulinemia can enhance the mitogenic effect of $\mathrm{Neu}$ oncogenic transformation on terminal end buds during mammary growth and development. The MMTV-c-Neu mouse model has been previously used to determine the effect of a high-fat diet (HFD) on mammary gland hyperplasia. Although this is a different model from ours, having a much longer tumor latency, it is interesting to note that either 5 or 10 weeks HFD treatment in FVB/ $\mathrm{N}$ mice had no effect on mammary gland hyperplasia in MMTV-c-Neu mice compared with controls, suggesting that at very early stages of tumor development, insulin may have a more potent effect than dietary fat in promoting terminal end bud hyperplasia. In the same model, it was shown that an HFD based on corn oil resulted in a shorter tumor latency than an HFD based on fish oils, suggesting that at later stages of tumor development, fats may play a role in tumor development but individual dietary components have different effects (Luijten et al. 2007).

We found that chronic (2-6 weeks) elevations of $\mathrm{Neu}$-NT led to significantly larger mammary tumors in MTB/TAN/MKR ${ }^{+/+}$mice compared with controls, suggesting that hyperinsulinemia enhances Neu-NT-mediated tumor formation and growth. This result is in agreement with our previous finding that orthotopic inoculation of three different mammary tumor cell lines (one overexpressing $\mathrm{Neu}$ ) each resulted in larger tumor formation in MKR mice compared with controls (Novosyadlyy et al. 2010, Ferguson et al. 2012) and demonstrates that $\mathrm{Neu}$-NT-mediated mammary tumor growth can be specifically enhanced by hyperinsulinemia. The mechanism by which insulin could increase tumor growth in the Neu-NT model may be complex. Our results demonstrate that MTB/TAN/MKR ${ }^{+/+}$mice demonstrate higher levels of phosphorylated IR/IGF1R than MTB/TAN control mice, suggesting that insulin is acting through its cognate receptor and/or the highly homologous IGF1R to promote mammary tumor growth. In human breast cancer tissues, phosphorylated IR/IGF1R has been reported to be a prognostic marker of poor outcome for breast cancer, regardless of subtype (Law et al. 2008). The ratio of IR isoform A (IR-A) to isoform B (IR-B) is also important in breast cancer, and recently, a higher IR-A:IR-B ratio has been observed in the luminal B subtype of breast cancer (Huang et al. 2011). Breast cancer cells in culture have also been reported to express higher levels of the IR (Osborne et al. 1978) and proliferate directly in response to insulin (Bollig-Fischer et al. 2011). The signaling pathways activated by the Neu-NT tyrosine kinase significantly overlap with those of the IR/IGF1R and include the canonical PI3-K/Akt/mTOR and MAPK signaling pathways. It is thus possible that activation of the IR/IGF1R causes amplification of the canonical Neu signaling pathways, which could possibly lead to increased tumor cell proliferation and/or survival.

Under conditions of hyperinsulinemia, IGF binding proteins (IGFBP)- 1 and -2 are repressed (Calle \& Kaaks 2004), which may lead to an increase in circulating 'free' IGF1. In the MTB/TAN/MKR ${ }^{+/+}$mouse model, increased IGF1 levels at the level of the target tissue could lead to increased activation of the IGF1R, thus directly increasing tumor growth. The Neu RTK has no known ligand, depending on its activation upon dimerization with either ErbB3 or ErbB1 (Cho et al. 2003, Garrett et al. 2003). Interestingly, $\mathrm{Neu}$ also dimerizes with IGF1R, an occurrence that provides a significant source of resistance to Her2-mediated therapies in humans (Lu et al. 2001, 2004). Thus, in our MTB/TAN/MKR ${ }^{+/+}$mouse model, it is possible that elevations of either insulin or 'free' IGF1 could promote tumor growth through increased activation of Neu/IGF1R hybrids (Nahta et al. 2006).

Our finding of greater numbers of lung metastases in MTB/TAN/MKR ${ }^{+/+}$mice suggests that, as well as promoting primary tumor growth, insulin may also enhance primary tumor progression and/or circulating tumor cell survival in the lung. Our previous work has demonstrated that murine mammary tumor cell line Mvt1 is able to form more lung metastases in MKR mice as a result of whether an orthotopic cell inoculation or an intravenous injection (Ferguson et al. 2012). Evidence that the IR specifically is involved in either homing to or survival in the lung has come from a report by Zhang et al. (2010), who showed that shRNA knockdown of the IR in breast cancer cell line LCC6 resulted in reduced ability of these cells to form lung metastases following intravenous injection into nude mice. The EMT permits tumor cells to gain the plasticity

Published by Bioscientifica Ltd. 
required to extravasate from the primary tumor site. During extravasation cells become increasingly mesenchymal in nature due to numerous changes in cytoskeletal scaffolding protein structures. Vimentin belongs to the intermediate filament (IF) family of proteins and has recently been shown to be an important marker of the EMT in epithelial cells that normally express only cytokeratin-type IFs (Zeisberg \& Neilson 2009, Satelli \& Li 2011). Vimentin is not specific for EMT and is also a marker of cancer-associated fibroblasts (Sugimoto et al. 2006). Additionally, recent studies have demonstrated that cancer-associated fibroblasts induce EMT in breast cancer cells (Soon et al. 2013). In breast cancer cells, vimentin expression has been shown to be correlated with increased migration and invasion (Gilles et al. 2003, Korsching et al. 2005), and in human breast cancer specimens, several studies have reported overexpression of vimentin as a marker of poor prognosis (Kokkinos et al. 2007). In this study, we observed elevated expression of vimentin as early as 6 weeks of Neu-NT upregulation. The majority of the vimentin-positive cells did not co-stain with Neu. This suggests the MTB/TAN/MKR ${ }^{+/+}$mice have a greater desmoplastic reaction to the tumors, with more numerous cancer-associated fibroblasts. Another explanation that we cannot exclude is that these vimentin-positive cells are tumor cells that have undergone EMT and have lost Neu expression. Our finding of hyperinsulinemia leading to greater lung metastasis could thus be due to an increase in the dissemination of tumor cells from the primary tumor associated with increase in the number of cancerassociated fibroblasts, or due to a direct effect of insulin on the tumors, inducing EMT. It is also possible that hyperinsulinemia may lead to greater cell survival, intravasation, and proliferation in lung tissue.

In summary, we have shown that hyperinsulinemia promotes advanced mammary gland hyperplasia, primary tumor growth, and lung metastasis in a Her2/Neu model of breast cancer. Further studies are required to determine whether, in promoting lung metastases, hyperinsulinemia enhances cell dissemination from the primary tumor, cell survival/adhesion in the lung, or both of these processes.

\section{Declaration of interest}

The authors declare that there is no conflict of interest that could be perceived as prejudicing the impartiality of the research reported.

\section{Funding}

This work was supported by the NIH/NCI (RO1CA128798-01A3) and American Diabetes Association (1-13-BS-108).

\section{Acknowledgements}

The authors thank Lewis A Chodosh, MD, PhD (Perelman School of Medicine, University of Pennsylvania, PA, USA), for donating MMTVMTB/TAN transgenic mice. They also thank Dr Archana Vijayakumar for performing animal insulin measurements.

\section{References}

Baselga J \& Swain SM 2010 CLEOPATRA: a phase III evaluation of pertuzumab and trastuzumab for HER2-positive metastatic breast cancer. Clinical Breast Cancer 10 489-491. (doi:10.3816/ CBC.2010.n.065)

Bollig-Fischer A, Dewey TG \& Ethier SP 2011 Oncogene activation induces metabolic transformation resulting in insulin-independence in human breast cancer cells. PLOS ONE 6 e17959. (doi:10.1371/journal.pone. 0017959)

Boyle P, Boniol M, Koechlin A, Robertson C, Valentini F, Coppens K, Fairley LL, Zheng T, Zhang Y, Pasterk M et al. 2012 Diabetes and breast cancer risk: a meta-analysis. British Journal of Cancer 107 1608-1617. (doi:10.1038/bjc.2012.414)

Calle EE \& Kaaks R 2004 Overweight, obesity and cancer: epidemiological evidence and proposed mechanisms. Nature Reviews. Cancer 4 579-591. (doi:10.1038/nrc1408)

Cho HS, Mason K, Ramyar KX, Stanley AM, Gabelli SB, Denney DW, Jr \& Leahy DJ 2003 Structure of the extracellular region of HER2 alone and in complex with the Herceptin Fab. Nature 421 756-760. (doi:10.1038/ nature01392)

Duggan C, Irwin ML, Xiao L, Henderson KD, Smith AW, Baumgartner RN, Baumgartner KB, Bernstein L, Ballard-Barbash R \& McTiernan A 2011 Associations of insulin resistance and adiponectin with mortality in women with breast cancer. Journal of Clinical Oncology 29 32-39. (doi:10.1200/JCO.2009.26.4473)

Ferguson RD, Novosyadlyy R, Fierz Y, Alikhani N, Sun H, Yakar S \& Leroith D 2012 Hyperinsulinemia enhances c-Myc-mediated mammary tumor development and advances metastatic progression to the lung in a mouse model of type 2 diabetes. Breast Cancer Research 14 R8. (doi:10.1186/bcr3089)

Fierz Y, Novosyadlyy R, Vijayakumar A, Yakar S \& LeRoith D 2010 Insulinsensitizing therapy attenuates type 2 diabetes-mediated mammary tumor progression. Diabetes 59 686-693. (doi:10.2337/db09-1291)

Formica V, Tesauro M, Cardillo C \& Roselli M 2012 Insulinemia and the risk of breast cancer and its relapse. Diabetes, Obesity \& Metabolism 14 1073-1080.

Garrett TP, McKern NM, Lou M, Elleman TC, Adams TE, Lovrecz GO, Kofler M, Jorissen RN, Nice EC, Burgess AW et al. 2003 The crystal structure of a truncated ErbB2 ectodomain reveals an active conformation, poised to interact with other ErbB receptors. Molecular Cell 11 495-505. (doi:10.1016/S1097-2765(03)00048-0)

Gilles C, Polette M, Mestdagt M, Nawrocki-Raby B, Ruggeri P, Birembaut P $\&$ Foidart JM 2003 Transactivation of vimentin by $\beta$-catenin in human breast cancer cells. Cancer Research 63 2658-2664.

Goodwin PJ, Ennis M, Pritchard KI, Trudeau ME, Koo J, Madarnas Y, Hartwick W, Hoffman B \& Hood N 2002 Fasting insulin and outcome in early-stage breast cancer: results of a prospective cohort study. Journal of Clinical Oncology 20 42-51. (doi:10.1200/JCO.20.1.42)

Goodwin PJ, Ennis M, Pritchard KI, Trudeau ME, Koo J, Taylor SK \& Hood N 2012 Insulin- and obesity-related variables in early-stage breast cancer: correlations and time course of prognostic associations. Journal of Clinical Oncology 30 164-171. (doi:10.1200/JCO.2011.36.2723)

Gunter MJ, Hoover DR, Yu H, Wassertheil-Smoller S, Rohan TE, Manson JE, Li J, Ho GY, Xue X, Anderson GL et al. 2009 Insulin, insulin-like growth factor-I, and risk of breast cancer in postmenopausal women. Journal of the National Cancer Institute 101 48-60. (doi:10.1093/jnci/djn415) 
Harari D \& Yarden Y 2000 Molecular mechanisms underlying ErbB2/HER2 action in breast cancer. Oncogene 19 6102-6114. (doi:10.1038/sj.onc. 1203973)

He X, Esteva FJ, Ensor J, Hortobagyi GN, Lee MH \& Yeung SC 2012 Metformin and thiazolidinediones are associated with improved breast cancer-specific survival of diabetic women with HER2 + breast cancer. Annals of Oncology 23 1771-1780. (doi:10.1093/annonc/mdr534)

Holbro T, Beerli RR, Maurer F, Koziczak M, Barbas CF, III \& Hynes NE 2003 The ErbB2/ErbB3 heterodimer functions as an oncogenic unit: ErbB2 requires ErbB3 to drive breast tumor cell proliferation. PNAS 100 8933-8938. (doi:10.1073/pnas.1537685100)

Huang J, Morehouse C, Streicher K, Higgs BW, Gao J, Czapiga M, Boutrin A, Zhu W, Brohawn P, Chang Y et al. 2011 Altered expression of insulin receptor isoforms in breast cancer. PLOS ONE 6 e26177. (doi:10.1371/ journal.pone.0026177)

Irwin ML, Duggan C, Wang CY, Smith AW, McTiernan A, Baumgartner RN, Baumgartner KB, Bernstein L \& Ballard-Barbash R 2011 Fasting $\mathrm{C}$-peptide levels and death resulting from all causes and breast cancer: the health, eating, activity, and lifestyle study. Journal of Clinical Oncology 29 47-53. (doi:10.1200/JCO.2010.28.4752)

Jin Q \& Esteva FJ 2008 Cross-talk between the ErbB/HER family and the type I insulin-like growth factor receptor signaling pathway in breast cancer. Journal of Mammary Gland Biology and Neoplasia 13 485-498. (doi:10.1007/s10911-008-9107-3)

Kokkinos MI, Wafai R, Wong MK, Newgreen DF, Thompson EW \& Waltham M 2007 Vimentin and epithelial-mesenchymal transition in human breast cancer - observations in vitro and in vivo. Cells, Tissues, Organs 185 191-203. (doi:10.1159/000101320)

Korsching E, Packeisen J, Liedtke C, Hungermann D, Wulfing P, van Diest PJ, Brandt B, Boecker W \& Buerger H 2005 The origin of vimentin expression in invasive breast cancer: epithelial-mesenchymal transition, myoepithelial histogenesis or histogenesis from progenitor cells with bilinear differentiation potential? Journal of Pathology 206 451-457. (doi:10.1002/path.1797)

Larsson SC, Mantzoros CS \& Wolk A 2007 Diabetes mellitus and risk of breast cancer: a meta-analysis. International Journal of Cancer $\mathbf{1 2 1}$ 856-862. (doi:10.1002/ijc.22717)

Law JH, Habibi G, Hu K, Masoudi H, Wang MY, Stratford AL, Park E, Gee JM, Finlay P, Jones HE et al. 2008 Phosphorylated insulin-like growth factor$\mathrm{I} /$ insulin receptor is present in all breast cancer subtypes and is related to poor survival. Cancer Research 68 10238-10246. (doi:10.1158/00085472.CAN-08-2755)

Lu Y, Zi X, Zhao Y, Mascarenhas D \& Pollak M 2001 Insulin-like growth factor-I receptor signaling and resistance to trastuzumab (Herceptin). Journal of the National Cancer Institute 93 1852-1857. (doi:10.1093/jnci/ 93.24.1852)

Lu D, Zhang H, Ludwig D, Persaud A, Jimenez X, Burtrum D, Balderes P, Liu M, Bohlen P, Witte L et al. 2004 Simultaneous blockade of both the epidermal growth factor receptor and the insulin-like growth factor receptor signaling pathways in cancer cells with a fully human recombinant bispecific antibody. Journal of Biological Chemistry 279 2856-2865. (doi:10.1074/jbc.M310132200)

Luijten M, Verhoef A, Dormans JA, Beems RB, Cremers HW, Nagelkerke NJ, Adlercreutz H, Penalvo JL \& Piersma AH 2007 Modulation of mammary tumor development in Tg.NK (MMTV/c-neu) mice by dietary fatty acids and life stage-specific exposure to phytoestrogens. Reproductive Toxicology 23 407-413. (doi:10.1016/j.reprotox.2006.12.001)

Moody SE, Sarkisian CJ, Hahn KT, Gunther EJ, Pickup S, Dugan KD, Innocent N, Cardiff RD, Schnall MD \& Chodosh LA 2002 Conditional activation of Neu in the mammary epithelium of transgenic mice results in reversible pulmonary metastasis. Cancer Cell 2 451-461. (doi:10.1016/S1535-6108(02)00212-X)

Nahta R, Yu D, Hung MC, Hortobagyi GN \& Esteva FJ 2006 Mechanisms of disease: understanding resistance to HER2-targeted therapy in human breast cancer. Nature Clinical Practice. Oncology 3 269-280. (doi:10.1038/ncponc0509)

Novosyadlyy R \& LeRoith D 2010 Hyperinsulinemia and type 2 diabetes: impact on cancer. Cell Cycle 9 1449-1450. (doi:10.4161/cc.9.8.11512)

Novosyadlyy R, Lann DE, Vijayakumar A, Rowzee A, Lazzarino DA, Fierz Y, Carboni JM, Gottardis MM, Pennisi PA, Molinolo AA et al. 2010 Insulinmediated acceleration of breast cancer development and progression in a nonobese model of type 2 diabetes. Cancer Research 70 741-751. (doi:10.1158/0008-5472.CAN-09-2141)

Osborne CK, Monaco ME, Lippman ME \& Kahn CR 1978 Correlation among insulin binding, degradation, and biological activity in human breast cancer cells in long-term tissue culture. Cancer Research $\mathbf{3 8}$ 94-102.

Peairs KS, Barone BB, Snyder CF, Yeh HC, Stein KB, Derr RL, Brancati FL \& Wolff AC 2011 Diabetes mellitus and breast cancer outcomes: a systematic review and meta-analysis. Journal of Clinical Oncology 29 40-46. (doi:10.1200/JCO.2009.27.3011)

Pinkas-Kramarski R, Shelly M, Glathe S, Ratzkin BJ \& Yarden Y 1996 Neu differentiation factor/neuregulin isoforms activate distinct receptor combinations. Journal of Biological Chemistry 271 19029-19032. (doi:10.1074/jbc.271.32.19029)

Pisani P 2008 Hyper-insulinaemia and cancer, meta-analyses of epidemiological studies. Archives of Physiology and Biochemistry 114 63-70. (doi:10.1080/13813450801954451)

Satelli A \& Li S 2011 Vimentin in cancer and its potential as a molecular target for cancer therapy. Cellular and Molecular Life Sciences 68 3033-3046. (doi:10.1007/s00018-011-0735-1)

Seshadri R, Firgaira FA, Horsfall DJ, McCaul K, Setlur V \& Kitchen P 1993 Clinical significance of HER-2/neu oncogene amplification in primary breast cancer. The South Australian Breast Cancer Study Group. Journal of Clinical Oncology 11 1936-1942.

Slamon DJ, Godolphin W, Jones LA, Holt JA, Wong SG, Keith DE, Levin WJ, Stuart SG, Udove J, Ullrich A et al. 1989 Studies of the HER-2/neu protooncogene in human breast and ovarian cancer. Science 244 707-712. (doi:10.1126/science.2470152)

Soon PS, Kim E, Pon CK, Gill AJ, Moore K, Spillane AJ, Benn DE \& Baxter RC 2013 Breast cancer-associated fibroblasts induce epithelial-to-mesenchymal transition in breast cancer cells. Endocrine-Related Cancer 20 1-12. (doi:10.1530/ERC-12-0227)

Sugimoto H, Mundel TM, Kieran MW \& Kalluri R 2006 Identification of fibroblast heterogeneity in the tumor microenvironment. Cancer Biology \& Therapy 5 1640-1646. (doi:10.4161/cbt.5.12.3354)

Ursini-Siegel J, Schade B, Cardiff RD \& Muller WJ 2007 Insights from transgenic mouse models of ERBB2-induced breast cancer. Nature Reviews. Cancer 7 389-397. (doi:10.1038/nrc2127)

Yarden Y 2001 Biology of HER2 and its importance in breast cancer. Oncology 61 (Suppl 2) 1-13. (doi:10.1159/000055396)

Zeisberg M \& Neilson EG 2009 Biomarkers for epithelial-mesenchymal transitions. Journal of Clinical Investigation 119 1429-1437. (doi:10.1172/JCI36183)

Zhang H, Fagan DH, Zeng X, Freeman KT, Sachdev D \& Yee D 2010 Inhibition of cancer cell proliferation and metastasis by insulin receptor downregulation. Oncogene 29 2517-2527. (doi:10.1038/ onc.2010.17)

Received in final form 27 March 2013

Accepted 9 April 2013

Made available online as an Accepted Preprint

9 April 2013 http://erc.endocrinology-journals.org DOI: 10.1530/ERC-12-0333
(C) 2013 Society for Endocrinology Printed in Great Britain 